\title{
Electrohydrodynamics of Charge Separation in Droplet-Based Ion Sources with Time-Varying Electrical and Mechanical Actuation
}

\author{
Thomas P. Forbes, ${ }^{a}$ F. Levent Degertekin, ${ }^{\mathrm{a}, \mathrm{b}}$ and Andrei G. Fedorov ${ }^{\mathrm{a}, \mathrm{b}}$ \\ ${ }^{a}$ G. W. Woodruff School of Mechanical Engineering, Georgia Institute of Technology, Atlanta, Georgia, USA \\ ${ }^{b}$ Parker H. Petit Institute for Bioengineering and Bioscience, Georgia Institute of Technology, Atlanta, \\ Georgia, USA
}

\begin{abstract}
Charge transport and separation in mechanically-driven, droplet-based ion sources are investigated using computational analysis and supporting experiments. A first-principles model of electrohydrodynamics (EHD) and charge migration is formulated and implemented using FLUENT CFD software for jet/droplet formation. For validation, classical experiments of electrospraying from a thin capillary are simulated, specifically, the transient EHD cone-jet formation of a fluid with finite electrical conductivity, and the Taylor cone formation in a perfectly electrically-conducting fluid. The model is also used to investigate the microscopic physics of droplet charging in mechanically-driven droplet-based ion sources, such as array of micromachined ultrasonic electrospray (AMUSE). Here, AMUSE is subject to DC and AC electric fields of varying amplitude and phase, with respect to a time-varying mechanical force driving the droplet formation. For the DC-charging case, a linear relationship is demonstrated between the charge carried by each droplet and an applied electric field magnitude, in agreement with previously reported experiments. For the AC-charging case, a judiciouslychosen phase-shift in the time-varying mechanical (driving ejection) and electrical (driving charge transport) signals allows for a significantly increased amount of charge, of desired polarity, to be pumped into a droplet upon ejection. Complementary experimental measurements of electrospray electrical current and charge-per-droplet, produced by the AMUSE ion source, are performed and support theoretical predictions for both DC- and AC-charging cases. The theoretical model and simulation tools provide a versatile and general analytical framework for fundamental investigations of coupled electrohydrodynamics and charge transport. The model also allows for the exploration of different configurations and operating modes to optimize charge separation in atmospheric pressure electrohydrodynamic ion sources under static and dynamic electrical and mechanical fields. (J Am Soc Mass Spectrom 2010, 21, 501-510) @ 2010 American Society for Mass Spectrometry
\end{abstract}

$I$ $\mathrm{n}$ the field of bioanalytical mass spectrometry (MS), the ion source generates and charges droplets/ particles of solvent/matrix containing the analyte molecules of interest. The capability of an ion source and its underlying ionization method to effectively disperse and charge analyte is a critical aspect that ultimately determines the efficiency of ionization. This, in turn, is among the key factors that determine the sensitivity of the mass spectrometric analysis. One of the most important ion sources for biomolecule ionization is electrospray ionization (ESI). ESI generates intact, low internal energy, gas-phase ions through the use of electrohydrodynamic formation and dispersion of a jet of electrically-conducting fluid under a strong applied electric field, known as a Taylor cone [1-3]. In this process, the electrically-conducting fluid is typi-

Address reprint requests to Prof. Andrei G. Fedorov, Woodruff School of Mechanical Engineering, Parker H. Petit Institute for Bioengineering and Bioscience, Georgia Institute of Technology, Atlanta, GA 30332-0405, USA. E-mail: andrei-fedorov@me.gatech.edu cally delivered through a thin capillary. As a strong electric field is applied, free charge migration to the liquid surface is induced. Charge accumulation at the surface creates electrical stresses that elongate the emerging fluid interface, producing a conical shape. The cone apex contains the highest charge density, where the electrical stresses overcome surface tension, emitting a liquid jet. In the setting of ESI-MS, the jet breaks up into highly charged droplets, eventually leading to desolvated ions.

Electrohydrodynamic atomization and tip streaming have been studied experimentally, analytically, and, more recently, computationally, in the field of mass spectrometry. Pioneering experimental work by Zeleny [4] and physical modeling by Taylor [5] considered the interface deformation experienced by an electricallyconducting fluid under electric fields. Over the years, numerous reports of experimental characterization and visualization of various electrospraying modes under a variety of conditions appeared in the literature [4-10]. 
In parallel, analytical and computational models of varying complexity and implementation techniques were being developed [6, 7, 11-20]. Taylor's initial electrostatic solution of an equilibrium liquid cone formed under application of an electric field considers a limiting case of perfectly conductive fluid. This resulted in what is now known as the classical "Taylor cone" with the jet apex angle of $98.6^{\circ}$ [5]. Similar results with perfectly conductive liquids have been demonstrated in the area of liquid metal ion sources (LMIS) [21-24]. While providing an important qualitative insight into the physics of electrospray, a perfectly-conducting fluid approximation is, however, unable to capture the conejet formation, commonly seen experimentally with typical MS solvents. To address this challenge, several improved models have been developed that incorporate a simplified set of electrohydrodynamic equations for a "leaky dielectric fluid," first introduced by Melcher and Taylor [16], and reviewed by Saville [18]. In a leaky dielectric model, the free charge within the bulk liquid is assumed to be zero (electro-neutrality condition) and all charges are concentrated at the fluidair interface. The free charge accumulated at the surface modifies the electric field and exerts normal and tangential Maxwell stresses at the interface, resulting in formation of the experimentally-observed cone-jet profiles [11, 13, 18-20]. The most comprehensive models solve a complete set of coupled equations for the fluid flow field, electric field, and charge transport [14, 18, 25], which is often required for a wide range of applications, besides electrospray ionization.

New ion sources, such as array of micromachined ultrasonic electrospray (AMUSE), have been recently developed that allow for decoupling of the charge separation and droplet formation. In contrast to conventional Taylor-cone-based ESI, in which fluid dispersion and droplet charging are intimately coupled, the AMUSE ion source allows one to utilize mechanical actuation for droplet generation and independentlycontrolled electrical actuation for droplet charging [2634]. Computational modeling would enable insight into the microscopic details of physicochemical phenomena, underlying analyte ionization in AMUSE, on a microsecond time scale. Further, the simulations would allow one to evaluate ion source design modifications instrumental to developing an improved design and optimal operation.

In this work, a computational model is developed, employing the full set of electrohydrodynamic and charge transport equations. The model is applied to investigate charge transport in atmospheric pressure MS ion sources based on electrospray and mechanicallydriven droplet ejection. For all cases analyzed, the simulated results are compared to the experimental data either from the literature (ESI) or our experiments (AMUSE). The paper is organized as follows. We begin with a concise description of a few specific aspects of the model, leaving a more descriptive presentation of the model formulation for the interested reader in the supplementary content, which can be found in the electronic version of this article. This is followed by a brief discussion of the EHD model application to several special cases, relevant to MS ionization, including the electrospray from a thin capillary and mechanicallydriven droplet-based analyte charging. For the former case (ESI), both the cone-jet formation for a fluid with finite electrical conductivity as well as the Taylor cone formation for a perfectly-conductive fluid are simulated and compared to models and experimental results from the literature. For the latter case (AMUSE), the EHD model is coupled to the fluid mechanics model of mechanically-driven droplet generation, and simulation results are compared to our own experiments with both DC- and AC-electric fields used for droplet charging. The paper concludes with a summary of key results and new insights revealed by simulations and experiments, including their implications and possible venues for improving the ion source design and charging/ ionization efficiency.

\section{Model Development and Implementation}

A full electrohydrodynamic model is utilized to investigate charge separation phenomena in various ion sources. Currently, there is no commercial code that is capable of fully modeling the electrohydrodynamic atomization process and includes all relevant physics. The computational fluid dynamics (CFD) package FLUENT [35] provides a powerful framework for modeling hydrodynamics of complex flows, including free surface flows and fluid interface evolution, but is unfortunately not equipped to solve the electric field or charge transport equations. FLUENT does, however, allow for the incorporation of additional transport equations and boundary conditions through the built-in heuristic of generalized transient advective-diffusive type equations for user-defined scalars (UDS) and userdefined functions (UDF), which must be hand-coded by a user using $\mathrm{C} / \mathrm{C}++$ language. In this work, we take advantage of these particular capabilities to develop and implement a set of UDSs for the electric potential evolution and charge transport, as well as UDFs for the charge and electric (Maxwell) stress boundary conditions at all interfaces. These UDSs and UDFs for each scalar field are then solved along with the basic hydrodynamic equations. The complete EHD model formulation, including the FLUENT implementation aspects, is discussed in the supplementary content.

\section{Modeling Methodology}

The model employs the volume of fluid (VOF) technique [36-41] for tracking interface evolution due to its applicability to free surface flows where interface breakup and coalescence are important. The basic idea of VOF is to retain the phase (volume of each phase) data in each cell of a fixed computational domain as a volume fraction of the $i$ th fluid, $\alpha_{i}$. The interface evolu- 
tion is tracked by advancing fluid volumes forward in time through the solution of an advection equation (eq S10). For computational cells defining the interface between phases, fluid properties are calculated as a volume-fractive-weighted average of the two phases. However, one drawback of the VOF technique for interface tracking is that it produces a "diffuse" (i.e., consisting of several computational cells) interface instead of a sharp inter-phase boundary. The main differences in various VOF implementations are the method of discretization of the volume fraction advection equation and interface reconstruction.

The simplest reconstruction scheme is the simplified line interface calculation (SLIC) [42], which defines the interface within each cell using a straight line parallel to one of the coordinate directions. The donor-acceptor scheme is an improved version of SLIC, but still results in an interface parallel to one of the coordinate directions [38]. Higher-resolution differencing schemes, such as compressive interface capturing scheme for arbitrary meshes (CICSAM) [43] and inter- $\gamma$ differencing scheme [44], have also been developed to compress the interface broadening. The VOF method of Youngs [45] is an accurate scheme in which the interface is approximated by a straight line at some linear slope such that the fractional fluid volume is conserved. Since the interface is no longer parallel to one of the coordinate directions, improved accuracy is achieved. The geometric reconstruction scheme, based on the method of Youngs, is used by FLUENT in our simulations to represent the interface between liquid and gas phases. The geometric reconstruction scheme uses a piecewise-linear approach to determine the face fluxes for the partially filled cells at the interface.

The resolution of the interface reconstruction is limited by the mesh size. Regions of fluid comparable to the mesh size cannot be accurately resolved [40]. The areas of coarse meshing, specifically those with radii of curvature on the scale of the mesh, are irresolvable by these methods. The piecewise-linear approach for interface reconstruction flattens high curvature regions, resulting in numerical artificial surface tension [39]. Interface reconstruction in thin filament regions, where the interface normal approximations are inaccurate, are also subject to an error due to artificial surface tension. In these instances, artificial surface tension can be reduced and the accuracy of the solution improved by decreasing the mesh size. In the limit of an infinitely refined mesh, the exact solution is reconstructed [39]. In this work, for accurate results a quadrilateral element mesh is used in all areas of the simulated domains where surface tension effects are thought to be important.

\section{Computational Results}

\section{Model Validation}

Two classical cases of electrospray, the cone-jet formation in a finite electrical conductivity fluid (Figure S1), and Taylor cone formation in a perfectly conductive fluid (Figure S2) are used to validate the developed EHD model and solution methodology by comparison to experimental and simulation results from the literature. In the case of a finite electrical conductivity liquid, the fluid-gas interface evolution during electrospraying from an axisymmetric capillary is considered, which is representative of a typical ESI scenario described in the literature. Figure S1 shows the forming cone-jet fluid profile as well as charge and electric potential distributions. The results of our simulations are qualitatively similar to many previously experimentally visualized and computationally simulated cone-jet profiles for a fluid of finite electrical conductivity [7, 12-14, 19, 20]. The ultimate outcome of this electrospray mode is a well-known and experimentally-observed tip streaming phenomenon, which is clearly seen in Figure S1. The simulations also produce jet breakup as seen in the Figure S1. A number of scaling laws for jet breakup have been developed for determining the drop size, typically based on fluid properties and relevant length scales $[6,11]$.

To display the versatility of our implementation of the electrohydrodynamic model, we also considered the limiting case of electrospraying a perfectly conducting fluid (Figure S2). Here, the free charges instantaneously redistribute themselves along the domain boundaries relative to all other time scales. The free charges realign themselves to exactly cancel the internal electric field within the bulk fluid [46, 47]. As shown in Figure S2, the evolving cone forms a $98.6^{\circ}$ angle as analytically predicted in Taylor's seminal work for a perfectly conductive fluid [5], as well as more in recent experimental results and numerical simulations [11, 17]. The Figure S2 inset displays the velocity field at the Taylor cone's tip, demonstrating the vortex formation as described by Shtern et al. [48] and experimentally observed by Hayati et al. [12]. Further details on the cone-jet and Taylor cone simulations can be found in the supplementary content.

\section{Mechanically-Driven Droplet Ejection}

With the predictive capabilities of the EHD model established, it is applied to the case of a mechanicallydriven, droplet-based ion source, such as AMUSE, with independently-controlled DC- and AC-electric bias for charging the analyte. The results of these simulations are compared with integral measurements of the AMUSE ion source performance from the mass spectrometric and charging experiments (electric current and charge-per-droplet versus applied electric field), which are reported in the last section of the paper.

AMUSE is a novel ion source which conceptually differs from classical electrospray ionization by decoupling analyte charging and droplet/ion formation. ESI ion sources leave little room for control of the charging process because the electric field drives fluid dispersion as well as charge transport/separation. In contrast, the AMUSE ion source enables independent control of the 
charge separating electric field by utilizing mechanical means (focused ultrasonic waves) for droplet ejection. This results in control over both ejection regime (i.e., discrete droplet to continuous jet) and droplet charging through localized charge separation. Obviously, this independence of mechanical and electrical stimuli control is not absolute, as the droplet-charging electric field also locally influences the mechanical aspects of the droplet ejection process due to the Maxwell stresses. Also, the electrical field applied to the piezoelectric transducer for its actuation has some effect on the charging electric field. Yet, since the electric and mechanical signals can be spatially localized in AMUSE (e.g., by using charging electrodes placed in the immediate vicinity of the ejection nozzles) the degree of their independent control is substantial for all practical purposes.

Figure S3 demonstrates the droplet evolution during the ejection process within an individual nozzle of the AMUSE array. Ejection is driven by the pumping action of a sinusoidal standing pressure wave $(1 \mathrm{MHz})$ generated by the piezoelectric transducer. Visualization and scaling of the ejection process, specific to AMUSE, have been described elsewhere as a balance between the periodic pressure forces, surface tension, and viscous stresses [32, 34]. Comprehensive accounts of physics governing droplet formation, including the effect of an electric field are available $[17,36]$. The complex interface evolution dynamics (Figure S3) induce electromechanical stresses, which in turn result in coupled charge transport and electric field gradients at the interface. These processes occur on the temporal ( $\sim$ microsecond) and spatial $(\sim$ micrometer) scales that are not readily accessible experimentally. However, with help of a carefully validated EHD model, the microscopic details of fluid flow, charge transport, and electric field can be computationally investigated during the droplet ejection, even within the nozzle orifice on the microsecond time scale.

Before final simulations, sensitivity studies have been performed to investigate sensitivity of numerical results on the mesh density, computational cell shape, and domain size [32]. The mesh density has been selected to minimize the numerical errors and artificial effects due to VOF interface tracking, as discussed in the preceding Modeling Methodology section, to accurately capture the advancing fluid interface during an ejection cycle. In the bulk domain, the radial and axial mesh density of 10 quadrilateral elements per $\mu \mathrm{m}$ in the vicinity of the nozzle orifice is employed. This mesh density decreases to five elements per $\mu \mathrm{m}$ in the axial direction approaching the outlet of the computational domain (Figure S4). Sensitivity studies of the simulation domain size indicates that its radial extent greater than three orifice diameters and its axial length capturing two wavelengths (droplet-to-droplet) of the ejection cycle are sufficient to yield no observable differences in results.
The charge transport equations (eq S2) are solved for both positive and negative charge carriers. The charge carriers considered are ions introduced throughout the fluid domain in a spatially uniform (isotropic analyte charging) manner as an initial condition. Specifically, these are considered to be the ions introduced by adding a small amount, $0.1 \%$ (vol/vol), of acetic acid to an aqueous solvent (hydrogen cations and acetate anions). Relative to the timescales of relevant phenomena considered here, dissociation of the acid molecules is essentially instantaneous and the recombination of ions is neglected. Unipolar ion injection is not considered, but reduction of ions is accounted for to maintain an electro-neutral condition within the bulk fluid. (As consecutive droplets are ejected, thus removing net charge from the bulk, an electrochemical "reduction" condition is incorporated into simulations to maintain electroneutrality in the bulk reservoir.)

Simulating charge transport under the application of a DC electric field induces charge separation throughout the fluid domain. Positive hydrogen cations migrate toward the evolving droplet interface, increasing the net positive charge on ejected droplets (Figure 1). Hydrogen cations, having an electrical mobility approximately an order-of-magnitude larger than the acetate anions, for the most part experience transport dominated by migration. Through parametric investigations, it is determined that at low bulk charge density levels $\left(\sim 10^{1} \mathrm{C} / \mathrm{m}^{3}\right)$, those associated with purely aqueous solutions and the electric field magnitudes utilized here, the mechanical ejection process (i.e., fluid transport) is much faster than charge transport. This results in weak droplet charging because droplets are ejected before sufficient charge separation can occur. However, for high bulk charge levels $\left(\sim 10^{4} \mathrm{C} / \mathrm{m}^{3}\right)$, the mechanical ejection and charge transport processes take place on comparable timescales, thereby coupling the associated phenomena. All simulation results shown hereafter are for the high bulk charge levels, which are the most interesting for bioanalytical MS. Initial charge densities are determined by a $0.1 \%(\mathrm{vol} / \mathrm{vol})$ acetic acid addition to an liquid. This is also the solvent used in our recently reported AMUSE-MS charge separation experiments [29], as well as in the electric current and droplet charging measurements we report later in this paper, thus allowing for direct comparisons between the model predictions and experimental measurements.

Figure 1 shows the interface profile and pressure distribution for a time instant during the ejection cycle in which a single drop has pinched off and a second drop is evolving. The simulations show that the electric potential distribution is approximately linear except in the droplet region. Here, the electric field magnitudes are elevated above the driving electric field between the bias (charging) and counter electrodes due to the highlycharged, elongated fluid interface. As expected, the highest levels of charge accumulation are in the areas of the highest electric field strength, at the poles of the droplet and within the evolving interface. For the case 


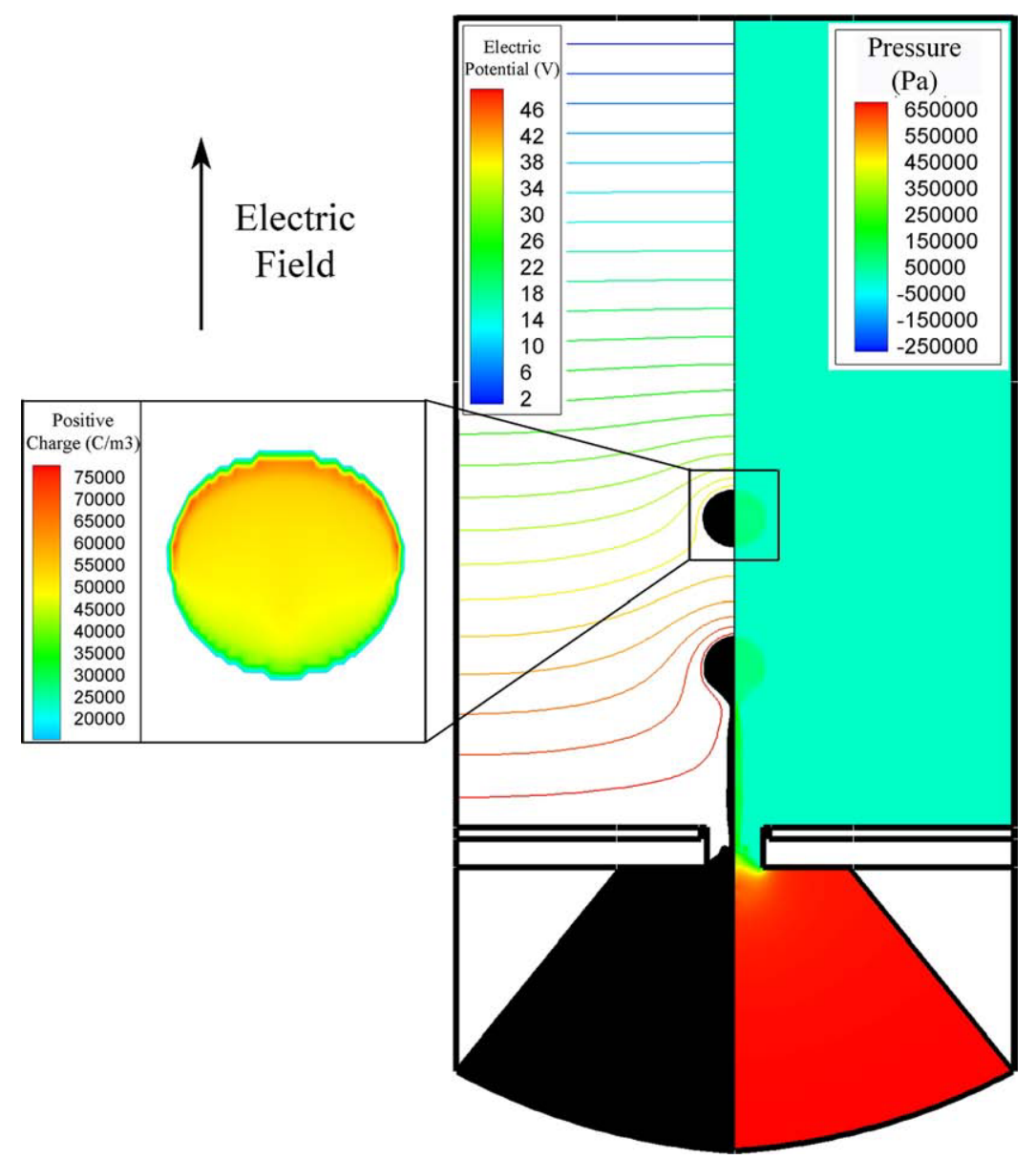

Figure 1. DC charging simulation results for electric potential distribution (left panel), pressure distribution (right panel), and a magnified view of positive charge distribution within an ejected droplet (inset). (Interface position is at liquid volume fraction equal to 0.5 ).

of applying a DC electric field, after a relatively quick transient, the bulk fluid maintains an approximately uniform potential (Figure 1). This reduces the electric field strength and, therefore, the charge migration, in the necking region of the evolving interface (Figure S3 (1-4). These phenomena will be discussed again when AC charging is described. However, as the drop pinches off (Figure S3 $(5,6)$, there is sufficient charge migration into the nozzle apex (orifice) region to produce increasingly charged droplets (Figure 1 inset).

Under the application of a DC electric field, charge separation occurs much as expected. Next, we investigate charge separation under a time-varying charging electric potential (field), specifically using a sinusoidal $\mathrm{AC}$ electric charging signal at the same frequency as the mechanical drive signal that generates the pressure field. The AC charging simulations use a "truncated" domain (see discussion in supplementary content) to improve the speed of calculations required for parametric analysis. All other aspects of the model implementation are identical to the "full" domain simulations for the DC charging case presented above. The initial simulation of droplet charging with an AC electric field, in-phase with the oscillating mechanical (pressure) field driving droplet ejection, yields net positive charge in ejected droplets (Figure 2). For the most part, the charge transport characteristics under the application of an AC electric field are similar to those discussed for the DC electric field simulations. However, with a continuously changing electric field, the bulk fluid never achieves a steady uniform potential. Comparing the electric potential distributions for DC (Figure 1) and AC (Figure 2) charging cases, there are obvious differences in the neck region of the evolving interface. Unlike the DC electric field, the applied AC electric field maintains an electric field presence in the necking region, allowing for increased migration of charge. This interesting theoretical result can be exploited in practice for improved droplet charging in mechanically-driven droplet-based ion sources, and we revisit this point in following sections.

With nearly-independent control of the mechanical (pressure) and electric fields, the phase shift between the ejection and charging signals can be varied to achieve either net positive or negative droplets. Figure 2 inset demonstrates the differences in the electric potential and net charge distributions for the pressure and electric fields in-phase (left) and $180^{\circ}$ out-of-phase (right). The electric field drives net charge to the far 


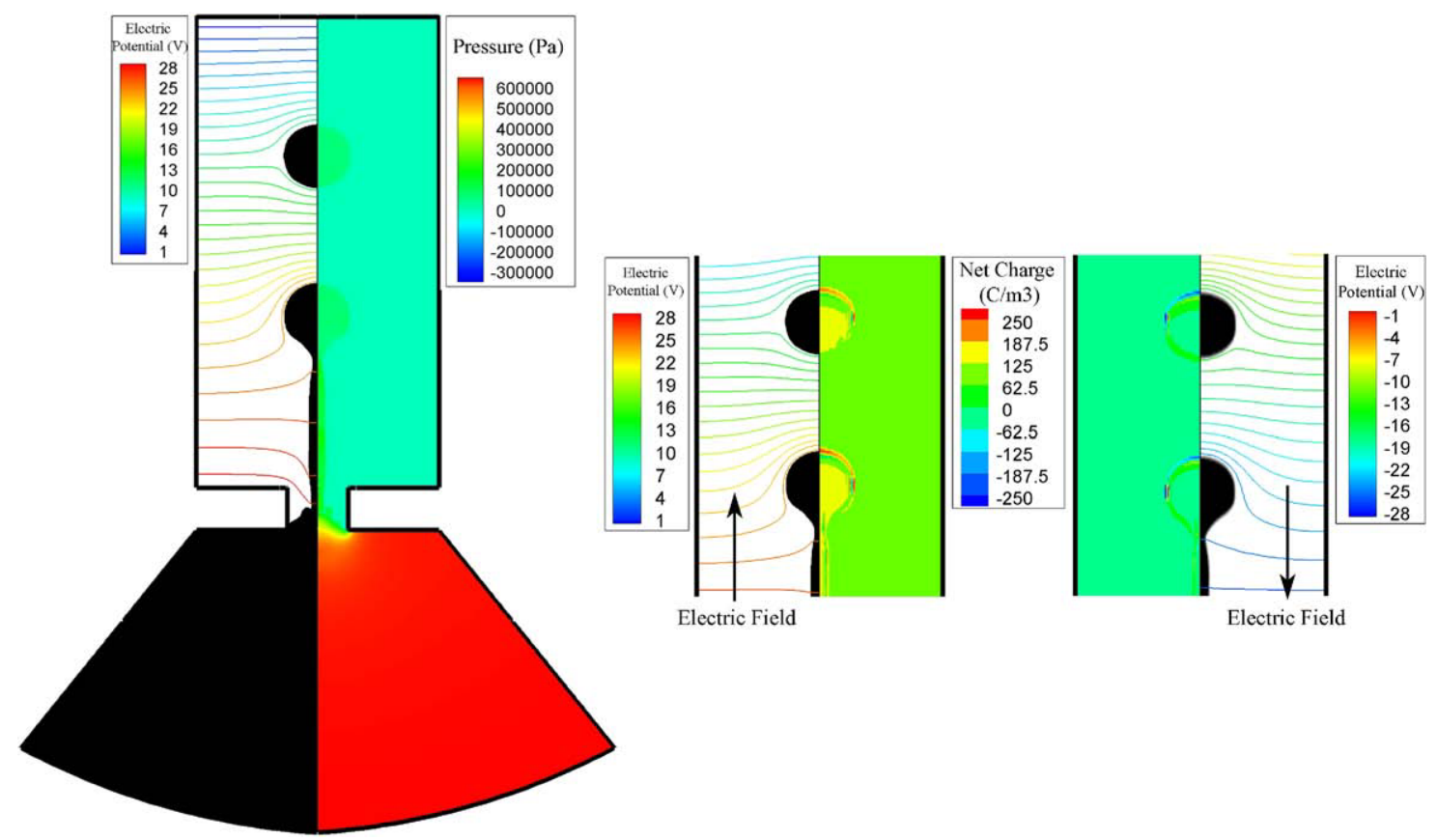

Figure 2. AC charging simulation results for electric potential distribution (left panel), pressure distribution (right panel), and a magnified view of electric potential and net charge distributions for electrical/mechanical signals in-phase (left inset) and $180^{\circ}$ out-of-phase (right inset). (Interface position is at liquid volume fraction equal to 0.5 ).

surface (north or south poles) of the ejected droplet, depending on the direction of the applied electric field. For the pressure and electric fields in-phase, as the electric field is positive (pointing toward the outflow from the nozzle), positive charges are also driven from the fluid bulk toward the orifice. The opposite is true for out-of-phase signals. The direction of the electric field during the time leading up to a droplet pinch-off determines the final net charge of ejected droplets. These unexpected trends in the case of AC charging are intriguing, and an experimental validation of the predictions on the effect of the phase shift between pressure and electric fields on droplet charging is presented in the next section.

\section{Experimental Results}

In the previous sections we have demonstrated the usefulness of the developed EHD model by considering three special cases, specifically, electrohydrodynamic cone-jet and Taylor cone formation, and charge transport in a mechanically-driven, droplet-based ion source (AMUSE). The computational predictions open up a window into the microscale physics of droplet charging in electrohydrodynamic ion sources. They also suggest a new way of using judiciously chosen AC fields for improved charge separation in the AMUSE ion source with independent control of electrical and mechanical actuation. In this last section, the results of theoretical predictions are compared qualitatively and quantitatively with experimental measurements on the AMUSE ion source with DC and AC charging.

\section{Experimental Setup}

To validate the trends and results obtained with the electrohydrodynamic simulations, a number of experiments measuring current and ejected mass (i.e., chargeper-droplet) have been carried out using an AMUSE ion source. Fabrication, assembly, and characterization of the AMUSE ion source have been described in detail elsewhere [26-34]. For the experiments in this study, the configuration equipped to apply a desired charging (bias) electrical potential, DC and AC, is used as described by Forbes et al. [29].

The experiments were conducted using an aqueous (deionized water; Ricca Chemical Company, Arlington, TX, USA) solution containing $0.1 \%$ (vol/vol) glacial acetic acid (BDH Aristar, Westchester, PA, USA) (pH 3.25). Schematics of the experimental setup used for DC and $\mathrm{AC}$ charging experiments are shown in Figure 3. In both cases, the piezoelectric transducer is driven by an amplified ( $\mathrm{T}$ and $\mathrm{C}$ Power Conversion rf Amplifier) rf signal at a resonant frequency of the fluid reservoir, generated by a Stanford Research Systems DS345 function generator labeled "rf Signal Generator 1" [26, 30, 34]. All respective signals were monitored with the use of a Tektronix TDS 2014 oscilloscope. The AMUSE ion source, operating under the specified electric field, ejected droplets into a small enclosed aluminum foil electrode box, collecting all ejected mass and charge. The current produced by the ejection of charged droplets was measured using a picoammeter (Keithley Instruments Inc., model 6485 picoammeter; Cleveland, $\mathrm{OH}, \mathrm{USA})$ as the droplets deposited their charge upon 

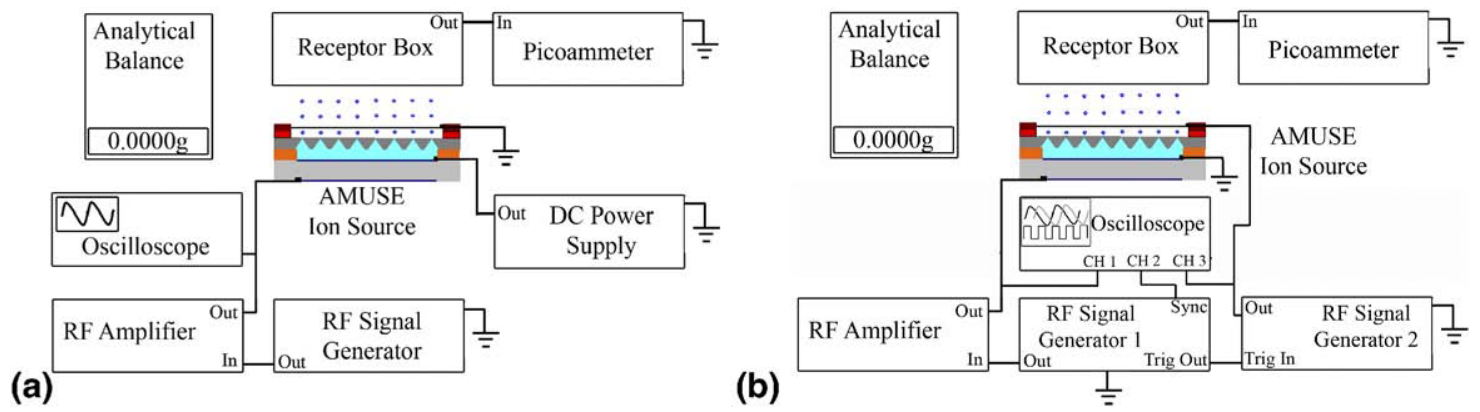

Figure 3. Experimental setup for ejected current and mass measurements for (a) DC charging and (b) AC charging with adjustable phase shift.

impingement on the metallic receptor. The typical environmental radio-frequency-induced noise was picked up by the sensitive picoammeter was $i_{R M S} \sim 0.05 \mathrm{nA}$. After a $60 \mathrm{~s}$ ejection period, the change in mass of the aluminum box was measured using an analytical scale (AE 200 Analytical Balance; Mettler-Toledo Inc., Columbus, OH, USA) with $0.1 \mathrm{mg}$ accuracy. An estimate of the charge-per-droplet was obtained by assuming uniform droplet size, with diameter approximately equal to that of the nozzle orifice [32]. Specific to the AC electric field experiments (Figure $3 b$ ), a second function generator, "rf Signal Generator 2" replaces the "DC Power Supply." The second function generator was triggered externally by "rf Signal Generator 1" to lock the phase of its signal to the signal from "rf Signal Generator 1" at the same frequency. All signals were monitored by the oscilloscope. The SRS DS345 function generators have phase shift adjusting capabilities to be used when considering AC charging mode of operation.

\section{Charging}

Figure 4a depicts the simulated charge-per-droplet as a function of applied DC electric field strength. As expected, a linear relationship is obtained, demonstrating the increase in charge-per-droplet with magnitude of external electric field. This is in agreement with the results of the MS-AMUSE droplet charging measurements as a function of external electric field [29]. Figure $4 \mathrm{~b}$ displays the charge-per-droplet for four consecutively ejected droplets, indicating a reduction in the "effective" charge-separating electric field acting on each consecutive droplet. This reduction is due to electric field shielding caused by previously ejected highly-charged droplets. The detrimental effect of shielding on droplet charging is unlikely to be an issue in typical mass spectrometry investigations using the AMUSE ion source, because of its coupling to a droplet transmission/evaporation interface (e.g., in-line air amplifier). The air amplifier immediately draws the droplets away from the ion source, transporting the droplet plume and improving desolvation before the mass spectrometer inlet [49].

For the range of electric field magnitudes analyzed in simulations, as well as employed in our previous and present experiments, the Maxwell stresses are not sufficient to produce significant interface deformation as seen in ESI. In electrohydrodynamic cone-jet atomization, much higher electric fields are utilized, which are further amplified at the capillary tip by the elongated
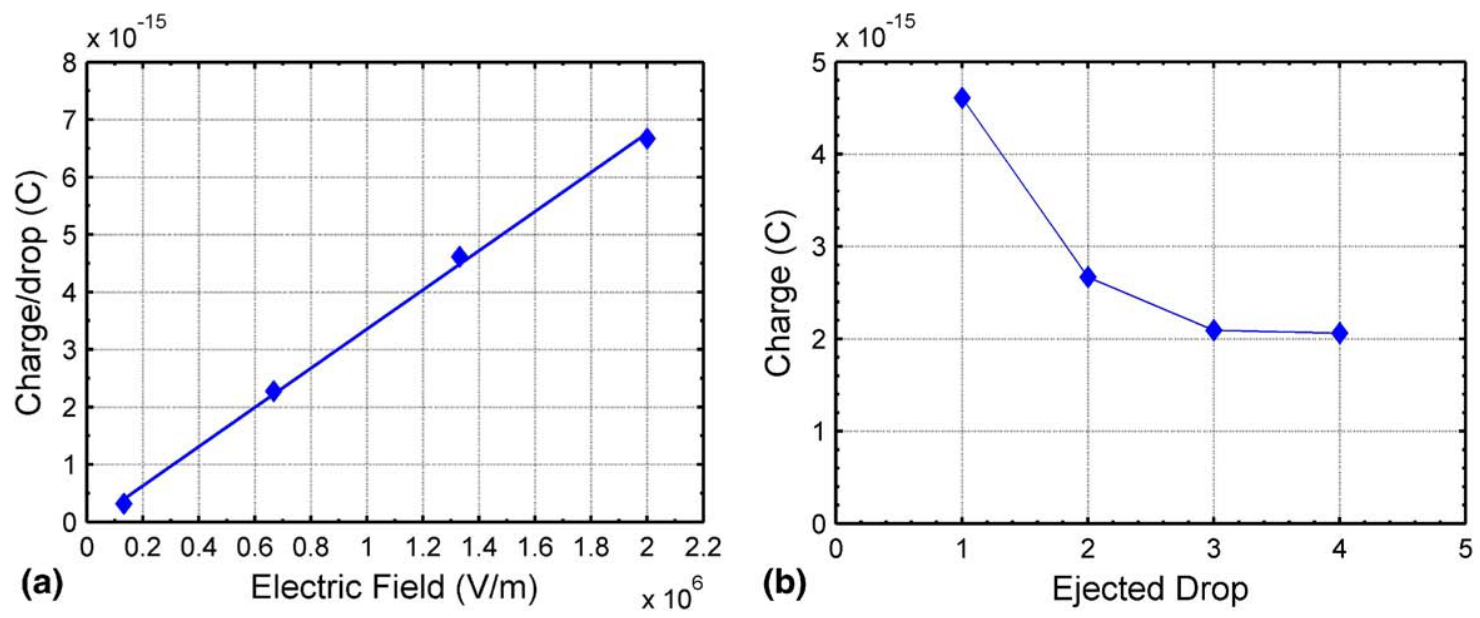

Figure 4. Simulated charge-per-droplet (a) as a function of DC charging electric field magnitude and (b) for consecutively ejected droplets. 
capillary geometry. Despite their rather low strength, the electric fields used in the AMUSE ion source are sufficient to increase the velocity of ejected highlycharged droplets by $\sim 50 \%$ across the range of electric field magnitudes considered in Figure 4a.

To validate these simulated trends, a number of experimental measurements were completed. Electric current data, in the range of nanoamperes to tens of nanoamperes, were taken for DC charging electric field magnitudes in the range previously used in our mass spectrometry measurements [29] and simulations. Figure 5 presents the results of experimental measurements and simulations, normalized for direct comparison. The circles represent the previously reported MS signal intensity data [29], obtained for a $3 \mu \mathrm{M}$ solution of reserpine in an aqueous solvent containing $0.1 \%$ (vol/vol) acetic acid, using a time-of-flight mass spectrometer (Bruker Daltonics micrOTOF; Billerica, MA, USA). The squares represent ejection current, measured using the setup in Figure 5a for an aqueous solvent with $0.1 \%$ acetic acid, and the diamonds represent the simulated charge-per-droplet as shown in Figure 6a. With linear fits to each set of data, we find remarkably similar results in the slope between experimental measurements and simulated results, providing validation of the trends produced by the model for the DC charging electric field.

To provide an approximate experimental measure of the charge-per-droplet, we coupled the electric current and ejected mass measurements. Ejected droplets were assumed to be monodisperse, with diameter approximately equal to the orifice diameter [32]. This provides a

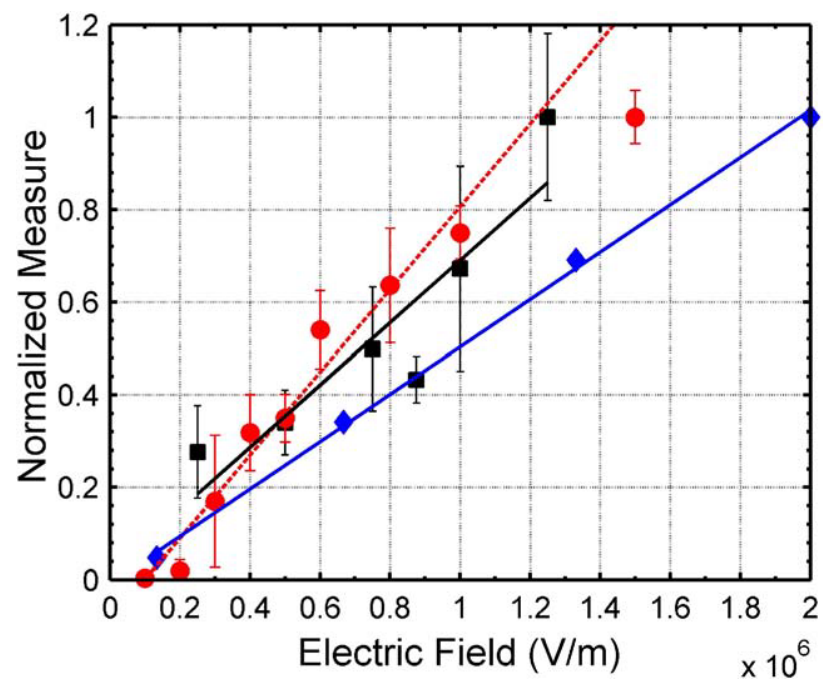

Figure 5. Dependence of normalized charge-per-droplet on the magnitude of the electric field-comparison between the experimental measurements and simulations. (Circles) Normalized mass spectrometry signal intensity [obtained for a $3 \mu \mathrm{M}$ solution of reserpine in an aqueous solvent containing $0.1 \%$ (vol/vol) acetic acid, using a time-of-flight mass spectrometer], (squares) normalized ejection current as measured in charge collection experiments described above [for an aqueous solvent with $0.1 \%$ (vol/vol) acetic acid], and (diamonds) normalized simulated charge-per-droplet. direct relationship between the mass of a single droplet and total mass collected during the experiment $(\sim 10-50$ $\mathrm{mg}$ per $60 \mathrm{~s}$ ). Experimental data on collected current and ejected mass yields estimates for charge-per-droplet in the range of $2 \times 10^{-15}-1 \times 10^{-14} \mathrm{C}$ across the range of $\mathrm{DC}$ electric field magnitudes considered here. Interesting to note, these charge-per-droplet measurements are of the same order-of-magnitude as the simulated results (Figure 4a) for equivalent electric fields. Also, at the highest electric field considered $\left(\sim 2 \times 10^{6} \mathrm{~V} / \mathrm{m}\right)$, both experimentally and computationally, the charge-per-droplet is an order-of-magnitude less than the Rayleigh limit for a water drop of similar size $\left(\sim 1 \times 10^{-13} \mathrm{C}\right)$.

\section{AC Charging}

With the use of a time-varying electric field, a great deal of control over charge separation and ultimately droplet charging can be gained. In this section, a special case is considered of AC charging at the same frequency as the acoustic pressure field to illustrate the power of judiciously-implemented interplay between the separately controlled mechanical and electrical fields, as a means to dramatically enhance the charging efficiency. The coupling between interface evolution, specifically pinch-off, and a dynamic electric field is a complex relationship, which, if well understood, can be exploited to optimize device operation and ionization efficiency.

Figure 6a shows simulated charge-per-droplet as a function of the relative phase-shift between the electric signal, which charges the droplets, and the acoustic pressure signal, which mechanically drives droplet ejection. As expected, a periodic sinusoidal relationship between ejected charge-per-droplet and the relative phase shift between acoustic pressure and electric charging signals is obtained. The simulated data points are plotted with a line to guide the underlying sinusoidal function. From the simulated net charge distributions plotted above (Figure 2 inset), positive charging is expected when the acoustic pressure field at the nozzle and electric charging signal are in-phase and negative charging when they are $180^{\circ}$ out-of-phase, exactly as shown in Figure 6a. To this end, the experimental charge measurements for an AC charge separating electric field are compared to the simulation results next.

The AC electrical field applied to the piezoelectric transducer to generate the acoustic pressure field also generates an electrical field with a fixed phase difference with respect to the pressure field. That fixed phase difference determines the polarity and amount of charge-per-droplet as simulated in Figure 6a, which can be called rf-only charging since there is no DC bias involved [44-46]. This can be considered as a constant, baseline charging level. To show that a separate AC electrical field can be used to control the droplet charging, a secondary AC signal at the same frequency is used. The secondary AC signal induces a charge- 

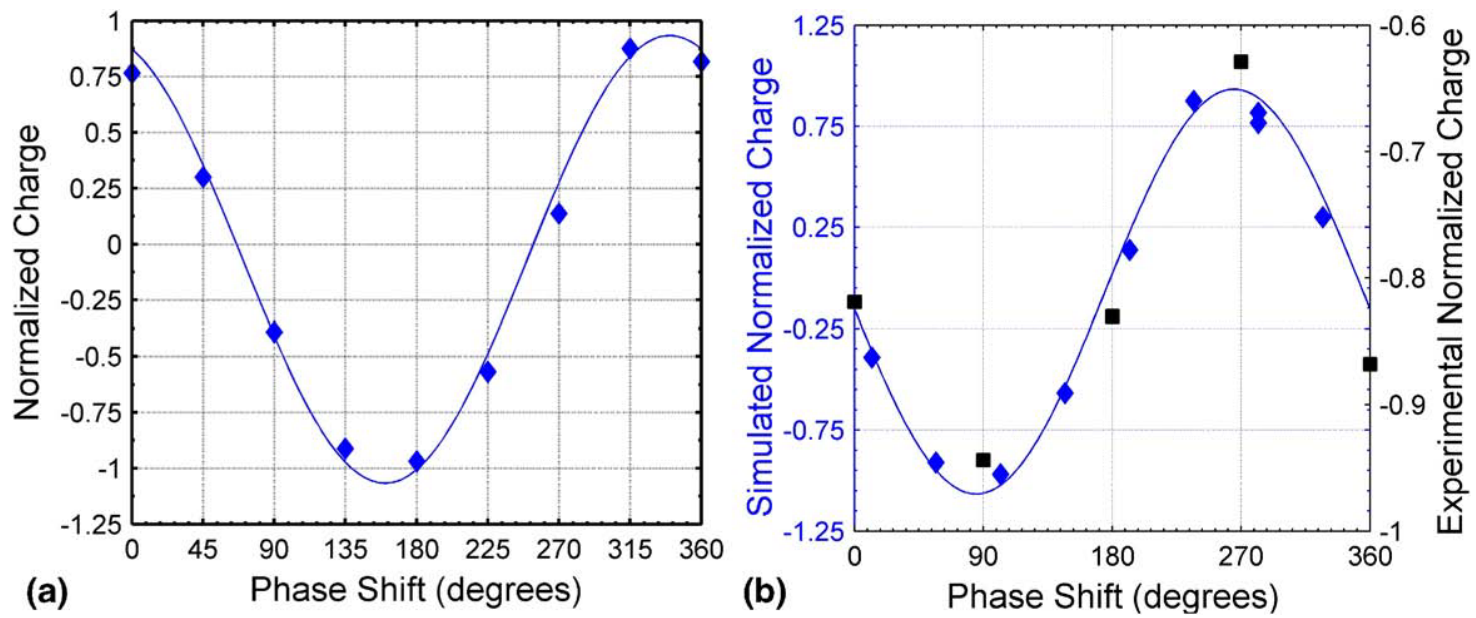

Figure 6. (a) Simulated charge-per-droplet as a function of the phase-shift between the AC charging electric field and mechanical ejection pressure field; (b) comparison of experimental normalized charge-per-droplet data with theoretical predictions as a function of a relative phase shift between the mechanical and electrical signals, (diamonds) CFD simulations of charge-per-drop, (squares) experimentally measured charge-per-drop using ejected current and collected mass measuring techniques described in the text.

separating electric field in the proximity of the nozzle at a desired phase shift with respect to the driving pressure signal. In such an arrangement, the secondary AC signal should introduce a modulation on the droplet charge over the baseline as this secondary electric field interferes with the electric field generated by the piezoelectric transducer drive signal (rf Signal Generators 1 and 2 in Figure $3 b$, respectively). Figure $6 b$ shows the experimental data and prediction of the charge-perdroplet as a function of a relative phase-shift, normalized for comparison. The experimental values are obtained from measurements of current and ejected mass, as a function of the phase-shift between the piezoelectric drive and secondary AC signals. The fixed phaseshift between pressure and electric signals generated by the piezoelectric transducers results in the net negative charge baseline, and the secondary electric field creates the expected sinusoidal relationship with maxima and minima in the measured charge-per-droplet. The secondary electric field interferes constructively and destructively with the fixed phase signal as the relative phase is varied between $0^{\circ}$ and $360^{\circ}$.

This result shows that a significant increase or a decrease in charge separation and droplet charging can be achieved by judicious combination of external DCand $\mathrm{AC}$-charging electric signals of appropriate waveform and phase-shift relative to the mechanical drive signal. This idea could be further extended to preferential pre-concentration of selected charged analyte molecules within an ejected droplet by exploiting the differences in ionic mobility in conjunction with the appropriately-chosen frequency, waveform, and phaseshift of the AC-charging electric field. The EHD model developed in this work becomes a powerful tool that makes such design exploration studies possible.

\section{Conclusions}

In this work, we report on the development and validation of an electrohydrodynamic computational model for atmospheric pressure ion sources with minimal simplifications. The model solves a coupled electrohydrodynamic problem for fluid flow, pressure and electrical potential fields, and charge transport in a multiphase fluidic system with evolving interfaces. The model is implemented using the FLUENT CFD platform and allows one to study a variety of complex phenomena involving interactions between the electrical and fluid mechanical fields.

We demonstrate the predictive capabilities of the model by successfully simulating the multiphysics processes for three exemplary cases relevant to analyte ionization in bioanalytical mass spectrometry. This includes prediction of the cone-jet structure in electrospraying a fluid with finite electrical conductivity, the quasi-equilibrium Taylor-cone formation in electrospraying an infinitely-conducting fluid, and effects of the DC- and AC-charging electric fields on charge separation and droplet charging in a mechanically-driven, droplet-based AMUSE ion source. The computational analysis predictions compared well with results of different charging experiments, flow visualization studies, and other simulations from the literature.

Lastly, we expect that the developed EHD model will serve as an enabling design tool, leading to exploration of new operating modalities for existing ion sources, especially those with significant degree of independent control of fluid dispersion and charge transport processes, such as AMUSE. The model also enables development of ideas for new types of ion sources, which allow for even greater control of the interacting fluid flow and charge transport phenomena. 


\section{Acknowledgments}

The authors thank Professor David Muddiman at North Carolina State University's W. M. Keck FT-ICR Laboratory for the informative and stimulating discussions. The authors also thank Dr. Peter Kottke at Georgia Institute of Technology for his technical help in developing the EHD model and thoughtful comments. The project described was supported by grant no. 1 R21 RR021474-01A1 from the National Center for Research Resources (NCRR), a component of the National Institutes of Health (NIH). Its contents are solely the responsibility of the authors and do not necessarily represent the official views of NCRR or NIH.

\section{Appendix A Supplementary Material}

Supplementary material associated with this article may be found in the online version at doi:10.1016/ j.jasms.2009.12.022.

\section{References}

1. Dole, M.; Mack, L. L.; Hines, R. L.; Mobley, R. C.; Ferguson, L. D.; Alice, M. B. Molecular Beams of Macro-Ions. J. Chem. Phys. 1968, 49(5), 2240-2249.

2. Fenn, J. B.; Mann, M.; Meng, C. K.; Wong, S. F.; Whitehouse, C. M. Electrospray Ionization for Mass Spectrometry of Large Biomolecules. Science 1989, 246(4926), 64-71.

3. Gaskell, S. J. Electrospray: Principles and Practice. J. Mass Spectrom. 1997, 32(7), 677-688.

4. Zeleny, J. Instability of Electrified Liquid Surfaces. Phys. Rev. 1917, 10(1), 1.

5. Taylor, G. Disintegration of Water Drops in an Electric Field. Proc. Royal Soc. London, Ser. A 1964, 280(1382), 383-397.

6. Gañán-Calvo, A. M.; Dávila, J.; Barrero, A. Current and Droplet Size in the Electrospraying of Liquids. Scaling laws. J. Aerosol Sci. 1997, 28(2), 249-275.

7. Hartman, R. P. A.; Brunner, D. J.; Camelot, D. M. A.; Marijnissen, J. C. M.; Scarlett, B. Electrohydrodynamic Atomization in the Cone-Jet Mode Physical Modeling of the Liquid Cone and Jet. J. Aerosol Sci. 1999, 30(7), 823-849.

8. Nemes, P.; Marginean, I.; Vertes, A. Spraying Mode Effect on Droplet Formation and Ion Chemistry in Electrosprays. Anal. Chem. 2007, 79(8), 3105-3116.

9. Marginean, I.; Nemes, P.; Parvin, L.; Vertes, A. How Much Charge is There on a Pulsating Taylor Cone? Appl. Phys. Lett. 2006, 89(6), 064104064103.

10. Parvin, L.; Galicia, M. C.; Gauntt, J. M.; Carney, L. M.; Nguyen, A. B.; Park, E.; Heffernan, L.; Vertes, A. Electrospray Diagnostics by Fourier Analysis of Current Oscillations and Fast Imaging. Anal. Chem. 2005, 77(13), 3908-3915.

11. Collins, R. T.; Jones, J. J.; Harris, M. T.; Basaran, O. A. Electrohydrodynamic Tip Streaming and Emission of Charged Drops from Liquid Cones. Nat. Phys. 2008, 4, 149-154.

12. Hayati, I.; Bailey, A.; Tadros, T. F. Investigations into the Mechanism of Electrohydrodynamic Spraying of Liquids: II. Mechanism of Stable Jet Formation and Electrical Forces Acting on a Liquid Cone. J. Colloid Interface Sci. 1987, 117(1), 222-230.

13. Hirt, C. W. Electrohydrodynamics of Semiconductive Fluids: With Applications to Electrospraying. Flow Sci. Technical Note 2004, 70, FSI-04-TN70.

14. Lastow, O.; Balachandran, W. Numerical Simulation of Electrohydrodynamic (EHD) Atomization. J. Electrostat. 2006, 64(12), 850-859.

15. Marginean, I.; Parvin, L.; Heffernan, L.; Vertes, A. Flexing the Electrified Meniscus: The Birth of a Jet in Electrosprays. Anal. Chem. 2004, 76(14), 4202-4207.

16. Melcher, J. R.; Taylor, G. I. Electrohydrodynamics: A Review of the Role of Interfacial Shear Stress. Annu. Rev. Fluid Mech. 1969, 1, 111-146.

17. Notz, P. K.; Basaran, O. A. Dynamics of Drop Formation in an Electric Field. J. Colloid Interface Sci. 1999, 213(1), 218-237.

18. Saville, D. A. Electrohydrodynamics: The Taylor-Melcher Leaky Dielectric Model. Annu. Rev. Fluid Mech. 1997, 29(1), 27-64.

19. Sen, A. K.; Darabi, J.; Knapp, D. R.; Liu, J. Modeling and Characterization of a Carbon Fiber Emitter for Electrospray Ionization. J. Micromech. Microeng. 2006, 16(3), 620.

20. Zeng, J.; Sobek, D.; Korsmeyer, T. Electro-Hydrodynamic Modeling of Electrospray Ionization: CAD for a $\mu$-Fluidic Device-Mass Spectrometer Interface. Proceedings of the Transducers '03 12th International Conference on Solid-State Sensors, Actuators, and Microsystems; Boston, MA, April; Digest of Technical Papers. 2003, 2, 1275-1278.

21. Barengolts, S.; Litvinov, E.; Suvorov, V.; Uimanov, I. Numerical Modeling of the Electrohydrodynamic and Thermal Instability of a Conducting Liquid Surface in a Strong Electric Field. Tech. Phys. Lett. 2001, 27(5), 370-372.

22. Suvorov, V. G. Numerical Analysis of Liquid Metal Flow in the Presence of an Electric Field: Application to Liquid Metal Ion Source. Surf. Interface Anal. 2004, 36(5/6), 421-425.

23. Suvorov, V. G.; Litvinov, E. A. Dynamic Taylor Cone Formation on Liquid Metal Surface: Numerical Modeling. J. Phys. D Appl. Phys. 2000, 33(11), 1245.

24. Suvorov, V. G.; Zubarev, N. M. Formation of the Taylor Cone on the Surface of Liquid Metal in the Presence of an Electric Field. J. Phys. D Appl. Phys. 2004, 37(2), 289.

25. Castellanos, A. Electrohydrodynamics, International Centre for Mechanical Sciences: Course and Lectures-No. 380. Springer: Wien, New York, 1998.

26. Aderogba, S.; Meacham, J. M.; Degertekin, F. L.; Fedorov, A. G.; Fernandez, F. M. Nanoelectrospray Ion Generation for High-Throughput Mass Spectrometry Using a Micromachined Ultrasonic Ejector Array. Appl. Phys. Lett. 2005, 86(20), 203110-203113.

27. Fedorov, A. G., Degertekin, F. L. Electrospray Systems and Methods; U.S. Patent no. 7,208,727.

28. Forbes, T. P.; Degertekin, F. L.; Fedorov, A. G. Multiplexed Operation of a Micromachined Ultrasonic Droplet Ejector Array. Rev. Sci. Instrum. 2007, 78(10), 104101-104106.

29. Forbes, T. P.; Dixon, R. B.; Muddiman, D. C.; Degertekin, F. L.; Fedorov, A. G. Characterization of Charge Separation in the Array of Micromachined Ultrasonic Electrospray (AMUSE) Ion Source for Mass Spectrometry. J. Am. Soc. Mass Spectrom. 2009, 20(9), 1684-1687.

30. Hampton, C. Y.; Forbes, T. P.; Varady, M. J.; Meacham, J. M.; Fedorov, A. G.; Degertekin, F. L.; Fernandez, F. M. Analytical Performance of a Venturi-Assisted Array of Micromachined Ultrasonic Electrosprays Coupled to Ion Trap Mass Spectrometry for the Analysis of Peptides and Proteins. Anal. Chem. 2007, 79(21), 8154-8161.

31. Hampton, C. Y. Silvestri, C. J.; Forbes, T. P. Varady, M. J.; Meacham, J. M.; Fedorov, A. G.; Degertekin, F. L.; Fernández, F. M. Comparison of the Internal Energy Deposition of Venturi-Assisted Electrospray Ionization and a Venturi-Assisted Array of Micromachined Ultrasonic Electrosprays (AMUSE). J. Am. Soc. Mass Spectrom. 2008, 19(9), 1320-1329.

32. Meacham, J. M. A Micromachined Ultrasonic Droplet Generator: Design, Fabrication, Visualization, and Modeling; Doctoral Dissertation, Georgia Institute of Technology, 2006.

33. Meacham, J. M.; Ejimofor, C.; Kumar, S.; Degertekin, F. L.; Fedorov, A. G. Micromachined Ultrasonic Droplet Generator Based on a Liquid Horn Sructure. Rev. Sci. Instrum. 2004, 75(5), 1347-1352.

34. Meacham, J. M.; Varady, M. J.; Degertekin, F. L.; Fedorov, A. G. Droplet Formation and Ejection from a Micromachined Ultrasonic Droplet Generator: Visualization and Scaling. Phys. Fluids 2005, 17(10), 100605100608.

35. FLUENT version 6.3, 2006. (FLUENT; Lebanon, NH).

36. Eggers, J. Nonlinear Dynamics and Breakup of Free-Surface Flows. Rev. Mod. Phys. 1997, 69(3), 865.

37. Harvie, D. J. E.; Fletcher, D. F. A New Volume of Fluid Advection Algorithm: The Defined Donating Region Scheme. Int. J. Numer. Methods Fluids 2001, 35(2), 151-172.

38. Hirt, C. W.; Nichols, B. D. Volume of Fluid (VOF) Method for the Dynamics of Free Boundaries. J. Comput. Phys. 1981, 39(1), 201-225.

39. Rider, W. J.; Kothe, D. B. Reconstructing Volume Tracking. J. Comput. Phys. 1998, 141(2), 112-152.

40. Rudman, M. Volume-Tracking Methods for Interfacial Flow Calculations Int. J. Numer. Methods Fluids 1997, 24(7), 671-691.

41. Rudman, M. A Volume-Tracking Method for Incompressible Multifluid Flows with Large Density Variations. Int. J. Numer. Methods Fluids 1998, 28(2), 357-378.

42. Noh, W. F.; Woodward, P. SLIC. (Simple Line Interface Calculations). Lecture Notes Phys. 1976, 59, 330-340.

43. Ubbink, O. Numerical Prediction of Two Fluid Systems with Sharp Interfaces; Ph.D. Thesis, Imperial College of Science, Technology, and Medicine, 1997.

44. Rusche, H. Computational Fluid Dynamics of Dispersed Two-Phase Flows at High Phase Fractions; Ph.D. Thesis, Imperial College of Science, Technology, and Medicine, 2002.

45. Youngs, D. L. Time-Dependent Multi-Material Flow with Large Fluid Distortion, Numerical Methods for Fluid Dynamics; Academic Press: London, 1982.

46. Griffiths, D. J. Introduction to Electrodynamics, 3rd ed.; Prentice Hall: NJ, 1999.

47. Landau, L. D.; Lifshitz, E. M.; Pitaevskii, L. P. Electrodynamics of Continuous Media, 2nd ed.; Elsevier Butterworth-Heinemann: Amsterdam, 2004.

48. Shtern, V.; Barrero, A. Striking Features of Fluid Flows in Taylor Cones Related to Electrosprays. J. Aerosol Sci. 1994, 25(6), 1049-1063.

49. Dixon, R. B.; Muddiman, D. C.; Hawkridge, A. M.; Fedorov, A. G. Probing the Mechanisms of an Air Amplifier Using a LTQ-FT-ICR-MS and Fluorescence Spectroscopy. J. Am. Soc. Mass Spectrom. 2007, 18(11), 1909-1913. 